\title{
Имитационное моделирование реверсивно-включаемых динисторов в режимах со сниженным порогом первичного запуска
}

\author{
(C) А.В. Горбатюк ${ }^{1, \uparrow, ~ Б . В . ~ И в а н о в ~}{ }^{2}$ \\ ${ }^{1}$ Физико-технический институт им. А.Ф. Иоффе Российской академии наук, \\ 194021 Санкт-Петербург, Россия \\ ${ }^{2}$ Санкт-Петербургский государственный электротехнический университет „ЛЭТИ“, \\ 197376 Санкт-Петербург, Россия \\ ฯ E-mail: agor.pulse@mail.ioffe.ru
}

(Получена 24 ноября 2016 г. Принята к печати 1 декабря 2016 г.)

Предложен и детально исследован методами имитационного моделирования новый способ включения реверсивно-включаемых динисторов (РВД) в субмикросекундные режимы с высокими скоростями нарастания коммутируемого тока при существенном снижении порога первичного запуска. В вычислительной задаче учитывались все значимые физические законы для пространственно-распределенных и дискретных элементов РВД-коммутатора, в том числе нелокальное изохронное взаимодействие между рабочими объемами реверсивно-включаемых динисторов или включающих фотодиодных оптронов и компонентами внешних цепей. Результаты моделирования подтвердили возможность практического достижения для реверсивно-включаемых динисторов скоростей нарастания тока вплоть до $d J / d t=3 \cdot 10^{10} \mathrm{~A} \cdot \mathrm{cm}^{-2} \cdot \mathrm{c}^{-1}$ в схемах на основе включающих полупроводниковых ключей малой мощности при пороге первичного запуска относительно реверсивно инжектированного заряда плотностью всего $1-2$ мкКл/см². Эти показатели ранее рассматривались только как теоретический предел, недостижимый в субмикросекундном диапазоне для реальных ключей тиристорного типа.

DOI: $10.21883 /$ FTP.2017.06.44565.8461

\section{1. Введение}

Многие приложения современной импульсной техники [1] нуждаются в разработках новых полупроводниковых приборов ключевого типа с характерной для тиристоров мощностью, но с существенно более высоким быстродействием. Среди потенциальных кандидатов особое внимание привлекают кремниевые реверсивновключаемые динисторы (РВД) [2-4], которые на протяжении десятилетий удерживают позицию наиболее мощных (до 1 ГВт на прибор) и надежных полупроводниковых переключателей микросекундного диапазона [5-9]. Однако попытки адаптировать РВД для коммутации субмикросекундных импульсов с крутыми фронтами нарастания тока сталкиваются с трудной проблемой необходимого увеличения интенсивности импульсов реверсивной накачки включающего заряда $Q_{R 0}$.

Количественной мерой достаточности по этому параметру, согласно теории [4], служит пороговая плотность $Q_{0}^{\text {cr }}$, пропорциональная задаваемой крутизне коммутируемых импульсов. В первых опытах по субмикросекундной коммутации РВД [10] необходимая величина $Q_{0}^{\text {cr }}$ оказалась столь высокой (до 150 мкКл/см ${ }^{2}$ ), что для технической реализации опытов на образцах площадью в сотые доли см ${ }^{2}$ потребовался громоздкий тиратронный генератор. В обзоре по схемотехническим принципам РВД [7] отмечалась возможность сокращения фронта включения ниже 1 мкс для конструкций с небольшим рабочим напряжением, $U \approx 1.5-2$ кВ, и тонкими базовыми слоями при оптимизированной форме управляющих импульсов.

Практически-рациональные субмикросекундные режимы работы низковольтных РВД в схеме с полу- проводниковыми управляющими элементами исследовались в работе [11]. Площадь опытных образцов составляла $0.5 \mathrm{~cm}^{2}$, а вычисляемая из осциллограмм скорость нарастания тока по плотности равнялась $d J_{A} / d t=2 \cdot 10^{9} \mathrm{~A} \cdot \mathrm{cm}^{-2} \cdot \mathrm{c}^{-1}$ при $Q_{R 0} \approx 20$ мкКл/см ${ }^{2}$. Это оказалось приемлемым для ряда пакетно-импульсных приложений, однако общие перспективы быстродействующих РВД оставались неясными из-за отсутствия в литературе необходимого для их оценки расчетнотеоретического базиса.

В работе [12], используя прецизионные методы компьютерного моделирования полупроводниковых приборов [13], мы исследовали субмикросекундную динамику уменьшенной по общей толщине конструкции РВД с буферным $n^{\prime}$-слоем в $n$-базе и двухслойным профилем диффузионного легирования их $p$-базы с уменьшенной до 10 мкм толщиной высоколегированной части с поверхностной концентрацией акцепторов $N_{p}^{s} \sim 10^{18} \mathrm{~cm}^{-3}$ и глубоким (50 мкм) подслоем со сниженным уровнем концентрации $N_{p}^{s} \sim 10^{16} \mathrm{~cm}^{-3}$. Рассчитанная крутизна импульсов, коммутируемых модифицированной конструкцией, оказалась во много раз более высокой, чем для высоковольтных РВД существующих типов. Однако при снижении плотности заряда накачки до нескольких единиц мкКл/см ${ }^{2}$ и „схемном“ сокращении коммутационных фронтов до 100-200нс на их расчетных характеристиках в интервале плотностей тока $\sim(300-500) \mathrm{A} / \mathrm{cm}^{2}$ неизбежно стали появляться высокие пики ,перенапряжения“ и аварийно-опасные задержки $\sim 10$ нс. В расчетах эти проявления исчезали при полном исключении глубокого $p$-слоя как элемента модели, но на практике такое решение может вызвать 
осложнения по части защиты РВД от краевого электрического пробоя.

Численный анализ [14] компромиссного варианта с двухслойной $p$-базой показал, что указанные нежелательные эффекты могут быть устранены при снижении концентрации $N_{p}^{s}$ до уровня $\sim 10^{15} \mathrm{~cm}^{-3}$. Скорость нарастания плотности тока после такой модификации составила $(2-3) \cdot 10^{10} \mathrm{~A} \cdot \mathrm{cm}^{-2} \cdot \mathrm{c}^{-1}$ при снижении управляющего заряда до 5-10 мкКл/см², что явилось новым расчетно-теоретическим пределом для субмикросекундных режимов РВД.

В недавнем сообщении [15] нами был предложен новый способ включения РВД, заключающийся в разбиении интервала импульсного управления на первичную стадию реверсивной накачки с током $J_{R}<0$ и на дополнительную стадию с положительным током $J_{G}>0$, в течение которой величина вводимого в динистор первичного заряда может быть доведена до более высокого уровня, необходимого для безопасной коммутации рабочего импульса тока. Поскольку этот эффект достигается благодаря внутренней тиристорной регенерации тока и за счет силового источника, порог первичного запуска, осуществляемого внешним управляющим генератором, может быть заметно снижен.

В настоящей работе мы детально исследуем физические особенности этого режима и изучаем возможность дальнейшего увеличения его эффективности при реализации в схеме РВД-коммутатора с полупроводниковыми управляющими ключами.

\section{2. Особенности запуска реверсивно-включаемого динистора в режимы с большой скоростью нарастания тока и их моделирование}

Базисные принципы РВД и основы их схемотехники были сформулированы и развиты в оригинальных публикациях разработчиков [2-7]. Оценку перспектив освоения РВД в субмикросекундном диапазоне можно дать на основе теории их порогового запуска [4], допускающей для случая малых толщин баз экстраполяцию на наносекундные начальные интервалы этапа формирования круто нарастающих импульсов. Так, в пренебрежении рекомбинацией избыточных зарядов для этих времен искомое условие достаточности по пороговой величине запускающего заряда принимает вид

$$
Q_{R 0} \geq Q_{R}^{\mathrm{cr}} \rightarrow B\left(w_{p}^{\mathrm{eff}}\right)^{4} d J_{A} / d t
$$

где $w_{p}^{\mathrm{eff}}-$ подгоночный параметр, соответствующий толщине сильнолегированной части $p$-базы с диффузионным переносом электронов, а $B \approx$ $\approx(5-10) \cdot 10^{-4} \mathrm{c}^{2} / \mathrm{cm}^{4}$ - размерный множитель, слабо зависящий от соотношения вкладов диффузии и дрейфа в динамику заряда $Q_{R 0}$ в слое его начальной локализации. Это условие удовлетворительно согласуется с

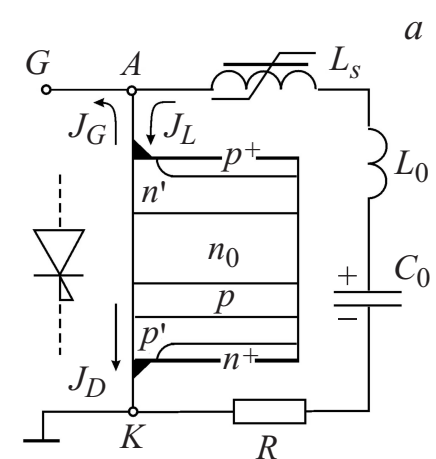

$b$

Рис. 1. Элементарная ячейка РВД в эквивалентной электрической цепи $(a)$ и иллюстрация идеи снижения порога его запуска $(b)$, где выделены управляющий, $J_{G}$, коммутируемый, $J_{L}$, и суммарный, $J_{D}$, импульсы тока; опорный режим обозначен как G-0, кривые для G-1 и G-2 помечены одним и двумя штрихами соответственно.

результатами работы [11] при $w_{p}^{\mathrm{eff}} \rightarrow 20$ мкм и объясняет достижение на порядок более высоких значений для крутизны коммутируемого тока в рассчитываемых образцах с модифицированным профилем легирования в [14].

Напомним некоторые особенности РВД, важные для нашей задачи „режимного“ облегчения реализации субмикросекундного запуска РВД. На рис. 1, a условно показаны рабочий объем РВД, представляющий собой интегральную совокупность большого числа, $k \sim\left(10^{3}-10^{4}\right)$, одинаковых элементарных шунтированных тиристорных ячеек (ЭТ), которые через общие катодный $(\mathrm{K})$ и анодный $(\mathrm{A})$ контакты соединены последовательно с дискретными элементами силовой цепи: емкостным накопителем $C_{0}$, нагрузкой $R$, паразитной индуктивностью $L_{0}$ и нелинейным дросселем с насыщающимся сердечником $L_{s}$. Нелинейный дроссель, индуктивность которого может меняться от исходно больших $\left(L_{s 0}\right)$ до малых конечных $\left(L_{s}\right)$ значений, является обязательным элементом РВД-коммутатора, обеспечивающим развязку управляющей и силовой цепей на время реверсивной накачки динистора и резкое последующее обострение фронта коммутируемого импульса.

В исходном состоянии емкость $C_{0}$ заряжена до напряжения $U_{0}$, индуктивность дросселя $L_{s} 0$ велика, а РВД находится в прямосмещенном запертом состоянии и блокирует разрядный ток. Для включения РВД через него от управляющего генератора $G$ в период индуктивной развязки цепей пропускают импульс тока реверсивной накачки $J_{R}(t)<0$. На дросселе в этот период возникает большое напряжение $U_{L 0}>U_{0}$, и через него начинает протекать слабо нарастающий ток, постепенно намагничивающий его сердечник к моменту $t_{s}$ до уровня насыщения и последующего перехода в состояние с малым $L_{s} 1{ }^{1}$

\footnotetext{
${ }^{1}$ Во временно́м масштабе всей задачи этот переход достаточно резок, и момент $t_{s}$ задается в расчетах как один из входных параметров наряду с $L_{s} 0$ и $L_{s} 1$.
} 
Из-за разных знаков контурных токов дросселя $J_{L}$ и импульса управления $J_{G}$ в период $0<t<t_{s}$ суммарный ток $J_{D}=J_{G}+J_{L}$ в некоторый момент $t_{R}$ может поменять знак с „-“ (на участке, где доминирует импульс $\left.J_{G}<0\right)$ на „,““, если начальная крутизна тока через дроссель достаточно велика (см. рис. $1, b)$. В этом случае реверсивная накачка РВД осуществляется только за счет отрицательной части импульса $J_{D}$ в интервале $t<t_{R}$.

Далее будем называть весь временной интервал формирования запускающего импульса от начала до момента насыщения дросселя, $0<t<t_{s}$, стадией $\mathbf{G}$, интервал реверсивного накопления включающего заряда $0<t<t_{R}-$ стадией $\mathbf{G}_{\mathbf{R}}$, а интервал коммутации рабочего импульса $t>t_{s}-$ стадией $\mathbf{F}$. Важно отметить, что появление вторичной „нереверсивной“ части с положительными мгновенными значениями в форме $J_{D}(t)-$ назовем ее стадией $\mathbf{G}_{\mathbf{F}}$ - открывает новую возможность оперативного регулирования интенсивности накачки при изменении амплитуды или длительности импульса $J_{G}$ (показано стрелками на рис. $1, b$ ).

Стандартное эмпирическое правило для запуска РВД состоит в таком согласовании всех параметров системы коммутатора, чтобы реверсивная накачка не прекращалась раньше момента обострения дросселя (т.е. часть $\mathbf{G}_{\mathbf{R}}$ должна полностью заполнять всю стадию $\left.\mathbf{G}\right)$. Этот вариант запуска РВД (далее - режим G-0, рис. 1, $b$ ) будет рассматриваться в последующих вычислениях как опорный для сравнения.

В случае выраженного изменения формы $J_{D}(t)$ при уменьшении амплитуды импульса $J_{G}(t)$ и большом вызываемом сокращении интервала $\mathbf{G}_{\mathbf{R}}$ становится возможной компенсация возникающего дефицита по управляющему заряду за счет расширения положительной части $\mathbf{G}_{\mathbf{F}}$ импульса $J_{D}(t)$ и дополнительно путем ,укручения“ медленной части импульса $J_{L}$ тока при соответствующем уменьшении величины $L_{s}$.

При этом результирующая величина накопленного заряда может быть увеличена до требуемого уровня достаточности к моменту $t_{s}$ при одновременном снижении порога первичного запуска. Определим этот модифицированный вариант запуска, идея которого была впервые сформулирована в работе [15], как режим G-1.

Наконец, здесь мы вводим в рассмотрение еще один возможный режим включения, в котором импульсы управления $J_{G}(t)$ принудительно укорачиваются так, чтобы $t_{G} \rightarrow t_{R} \ll t_{s}$ (см. рис. $\left.1, b\right)$. В этом случае вскоре после прекращения действия $J_{G}(t)$ импульс тока через РВД $J_{D}^{\prime \prime}(t)$ приближается по форме к импульсу тока через дроссель $J_{L}(t)$, а величина заряда, вводимого импульсом $J_{D}^{\prime \prime}(t)$ на „нереверсивной“ стадии управления $\mathbf{G}_{\mathbf{F}}$, может быть еще в разы увеличена по сравнению с вариантом G-1 даже при исходно малом $Q_{R 0}$. Этот режим включения, для практической реализации которого необходим „двухоперационный“ управляющий ключ, назовем режимом G-2.

Для сравнительной оценки эффективности режимов G-0, G-1 и G-2 мы выполнили несколько серий вы-
Таблица 1. Параметры структуры тиристорной ячейки

\begin{tabular}{c|c|c|c|c|c}
\hline Слои $\rightarrow$ & $n^{+}$ & $p^{\prime}$ & $p$ & $n^{\prime}$ & $p^{+}$ \\
\hline$N_{n, p}^{s}, \mathrm{~cm}^{-3}$ & $10^{20}$ & $10^{19}$ & $2 \cdot 10^{15}$ & $2 \cdot 10^{17}$ & $10^{19}$ \\
$w_{n, p}$, мкм & 3 & 7.5 & 50 & 12 & 4
\end{tabular}

числений на программном пакете SYNOPSYS [13]. При этом учитывались все транспортные эффекты диффузионно-дрейфового переноса, механизмы генерации-рекомбинации и ударной ионизации, эффекты сильного поля, высокого легирования и др. Считалось, как и ранее $[11,12,14]$, что все элементарные тиристорные ячейки (ЭТ) РВД физически эквивалентны. Это позволило рассматривать весь прибор как единичный двухэлектродный объемно-распределенный элемент, через который протекает $1 / k$-я часть тока подводящих контактов (рис. 1,a). Для единообразия интерпретации значения мгновенных токов через контакты РВД и параметры элементов цепей $R, L, C$ и др. были приведены к единице площади РВД.

Моделировалась структура с одинаковыми цилиндрическими ЭТ с радиусом $r_{m}=500$ мкм, высотой (толщиной пластины) $w=500$ мкм и радиусом шунтов $r_{0}=15$ мкм при исходном легировании толщи донорами $N_{n 0}=2 \cdot 10^{13} \mathrm{~cm}^{-3}$. Параметры донорных и акцепторных составляющих вертикальных диффузионных профилей легирования определены их поверхностными концентрациями $N_{n, p}^{s}$ и толщинами по глубине $w_{n, p}$ в табл. 1.

Для цепи нагрузки во всех трех сравниваемых вариантах задавались одинаковыми: напряжение $U_{C 0}=5 \mathrm{\kappa B}$, сопротивление нагрузки $R=0.5 \mathrm{OM} \cdot \mathrm{cm}^{2}$, емкость $C_{0}=$ $=313 \mathrm{H} \Phi / \mathrm{cm}^{2}$, паразитная индуктивность $L_{0}=50 \mathrm{H} \Phi \cdot \mathrm{cm}^{2}$, конечная индуктивность $L_{s 1}=50 \mathrm{H} \Phi \cdot \mathrm{cm}^{2}$ и задержка обострения дросселя $t_{s}=200$ нс. Выбор начальной индуктивности дросселя $L_{s} 0$ зависел от рассчитываемого варианта запуска.

Температура структуры в течение всего моделируемого периода считалась постоянной и равной $T=400 \mathrm{~K}$.

Импульсы управления в этой части работы задавались как явные зависимости $J_{G}=J_{G}(t)$ с формой, подобной показанной на рис. $1, b$, с фиксированной амплитудой и длительностью, а также с варьируемой величиной задержек нарастания, $\tau_{G}^{0}$, времен экспоненциального установления максимума, $\tau_{G}^{+}$, и экспоненциального спада, $\tau_{G}^{-}$, таким же образом, как и в работах $[14,15]$.

Результаты выполненных расчетов представлены на рис. 2, где сравниваются динамические характеристики РВД в классической функциональной схеме (рис. 1, $a$ ).

Сначала отметим особенности переходных характеристик для опорного режима G-0. Начальная индуктивность нелинейного дросселя была задана значением $L_{s 1}=4$ мкГн $\cdot$ см$^{2}$. Заданными параметрами импульса $J_{G}(t)$ были $t_{G}=200, \tau_{G}^{0}=20, \tau_{G}^{+}=\tau_{G}^{-}=50$ нс. Формы импульса тока через динистор $J_{D}$ (кривая 1 ) подбирались вариацией амплитуды $J_{G}$ таковой, чтобы нулевой 


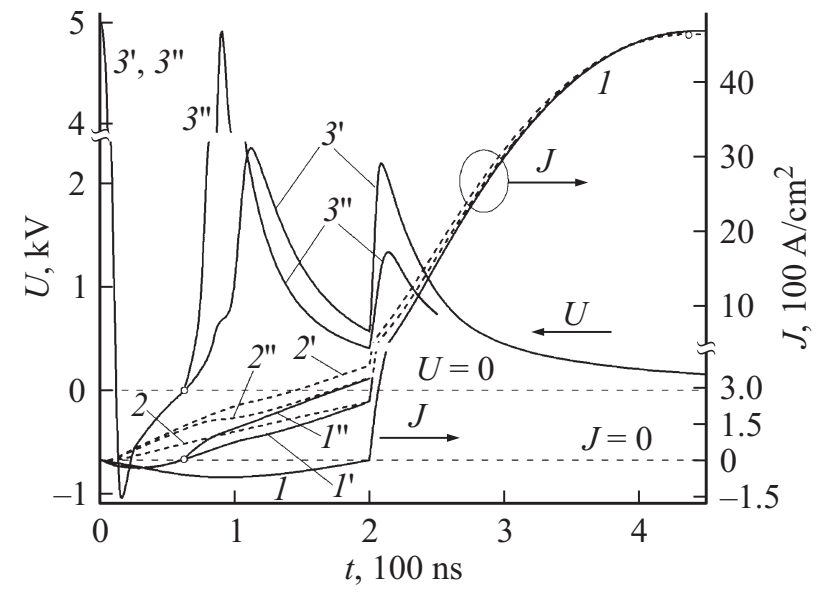

Рис. 2. Расчетные коммутационные характеристики РВД в режимах с заданной формой $J_{G}(t) .1-$ суммарный ток через динистор $J_{D}, 2$ - ток через элементы цепи нагрузки $J_{L}$, 3 - напряжение на аноде РВД $U_{A}$. Кривые для режимов G-1 и G-2 помечены штрихами в том же порядке, что и на рис. $1, b$.

уровень токов пересекался линией $J_{D}$ точно в момент насыщения дросселя $t_{s}$. Очевидно, что в этот период функция $U_{A}(t)$ отрицательна, а ток через дроссель (кривая 2) монотонно растет со временем. Для $t>t_{s}$ на расчетных характеристиках $U_{A}(t)$, как и в работе [14], в интервале первых 10 нс после момента обострения фронта и перехода на стадию быстрого нарастания тока $\mathbf{F}$ возникает одиночный переходный пик напряжения, происхождение которого обязано временно́й задержке установления „стационарного“ предела по механизму тиристорной регенерации тока.

Для модифицированных режимов G-1 и G-2 на кривой $U_{A}(t)$, кроме характерного (основного) пика на стадии $\mathbf{F}$, появляется высокий дополнительный пик на управляющей стадии $\mathbf{G}$. Если природа основного пика здесь идентична таковой для случая $\mathbf{G - 0}$, то появление дополнительного пика вызывается реакцией силового дросселя в состоянии с большой начальной индуктивностью $L_{s 0}$ на быструю смену полярности тока через динистор в момент $t_{R}<t_{s}$ (см. рис. $\left.1, b\right)$. Длительность импульса $J_{G}(t)$ задавалась равной $t_{G}=t_{s}$, но, в отличие от варианта G-0, теперь контролировался не сам момент $t_{R}$, а величина накапливаемого в этот период заряда $Q_{R}$, определяющая интенсивность первичного запуска. При одинаковых формах импульсов накачки $J_{G}(t)$ и, следовательно, равных значениях $Q_{R}$, основной пик для случая G-2 заметно снижается по сравнению с $\mathbf{G - 1}$, очевидно, благодаря большей величине $Q_{0}$, тогда как дополнительный пик увеличивается из-за относительного увеличения скорости роста импульса $J_{D}(t)$ при изменении его направления с отрицательного на положительное.

Скорость роста $J_{D}\left(t>t_{R}\right)$ для режима $\mathbf{G}-2$ зависит, очевидно, от времени спада $\tau_{G}^{-}$импульса генератора $J_{G}(t)$, увеличиваясь при его сокращении. В итоге, если при удлинении $\tau_{G}^{-}$в форме $U_{A}(t)$ должно наблюдаться асимптотическое сближение с вариантом G-1, то в противном случае высота переходного пика напряжения будет нарастать и в принципе сможет превысить порог начала лавинного пробоя, что, конечно, нуждается в отдельном исследовании.

Величины зарядов $Q_{G}$, накапливаемых в период управления, могут быть вычислены отдельно для „реверсивной“ $\left(\mathbf{G}_{\mathbf{R}}\right)$ и „нереверсивной“ $\left(\mathbf{G}_{\mathbf{F}}\right)$ стадий через интегральные формы по времени как

$Q_{G}^{-}=Q_{R}^{-}=\int_{0}^{t_{R}} J_{D} d t, Q_{G}^{+}=\int_{t_{R}}^{t_{s}} J_{D} d t, J_{D}=J_{G}(t)+J_{L}(t)$.

Здесь независимой функцией времени является только переменная $J_{G}(t)$, тогда как форма $J_{L}(t)$ диктуется моделируемой динамикой „плавающего“ потенциала анода $U_{A}(t)$ и должна извлекаться из полных численных решений.

В рамках нашей задачи основной интерес (с позиции реализуемости различных технических средств управления) представляет интенсивность первичной (реверсивной) накачки, которая должна быть достаточно высока по параметру $Q_{R}$, чтобы полностью исключить опасные проявления на внешних наблюдаемых характеристиках. С целью сравнения по $Q_{R}$ пики напряжения, детектируемые в ходе численного описания режимов G-0 и G-1, подбирались близкими по высоте и значительно меньшими относительно условного уровня $U_{C 0} / 2$, который из опыта наших расчетов в $[12,14,15]$ можно принять как разрешенный в смысле надежности. Рассчитанные величины зарядов, накапливаемых при реверсивной накачке в вариантах G-0, G-1, G-2, составили соответственно $Q_{R 0}^{(0)}=8.6$ и $Q_{R 0}^{(1)}=Q_{R 0}^{(2)}=1.27$ мкКл/см ${ }^{2}$. Отсюда следует, что с учетом принятых оговорок переход к модифицированному режиму G-1 позволит уменьшить порог надежного запуска субмикросекундного РВД почти в 7 раз относительно опорного варианта G-0 и тем самым ослабить требования к управляющему генератору.

В модифицированном варианте запуска G-2 высота дополнительного пика, появляющегося вскоре после смены знака $J_{D}(t)$, увеличивается при сокращении времени спада $\tau_{G}^{-}$внешнего управляющего импульса $J_{G}(t)$. На практике возможные значения $\tau_{G}^{-}$сильно зависят от типа выбираемого управляющего ключа. Здесь для определенности при расчете кривых $1^{\prime \prime}-3^{\prime \prime}$ время $\tau_{G}^{-}$было задано равным 25 нс. Как показало моделирование, при подгоночном снижении начальной индуктивности дросселя и сокращении длительности управляющих импульсов до $10-20$ нс параметр $Q_{R 0}^{(2)}$ может быть дополнительно уменьшен еще в 2-3 раза, вплоть до 0.4-0.8 мкКл/см². Подобные случаи, потенциально привлекательные для практики, конечно, требуют дополнительного изучения с точки зрения гарантий по эксплуатационной области безопасной работы относительно эффектов пробоя по такой же схеме, как было сделано в [16] для интегральных биполярных ключей. 

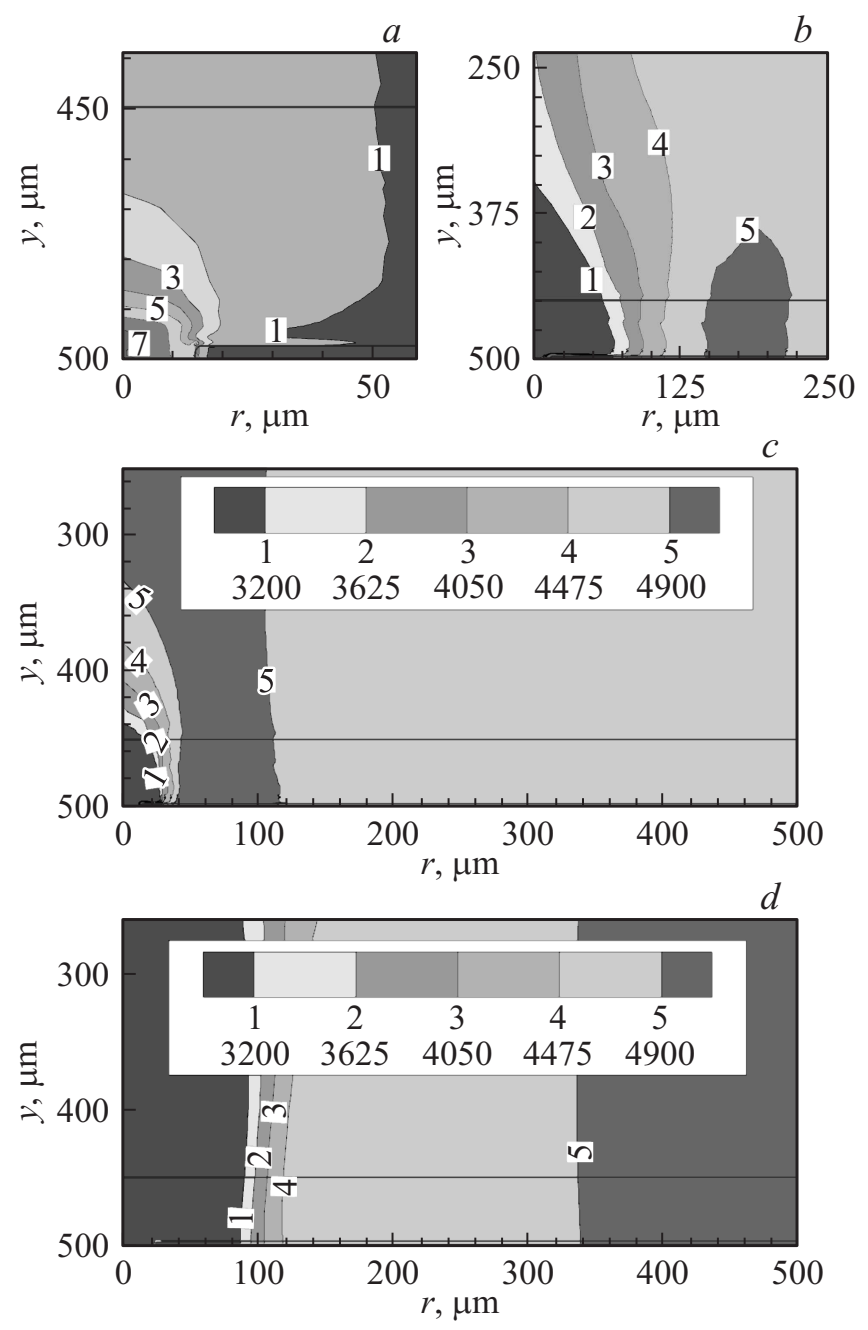

Рис. 3. Контурные линии уровней модуля плотности тока $|J(x, r)|$ в ячейках РВД для режимов G-0 $(a, c)$, G-1 $(b)$ и G-2 $(d)$ в моменты максимумов напряжения $t_{1}=208.8$ нс $(a, b)$ и тока $t_{2}=433 \mathrm{Hc}(c, d)$. $a$ : контуры $1-7$ соответствуют уровням от 0.5 (1) до 16.5 (7) с интервалом $2.5 \mathrm{\kappa A} / \mathrm{cm}^{2}$; $b$ : контуры $1-5$ соответствуют уровням от 180 (1) до 580 (5) с интервалом $100 \mathrm{~A} / \mathrm{cm}^{2} ; c, d$ : контуры $|J|$ и их номера обозначены на шкалах оттенков.

Заметим, что в полученном для варианта G-2 двумерном численном описании инжекционных процессов в РВД образование локализованных зон высокого поля со скоростью ударной ионизации $\sim\left(10^{22}-10^{23}\right) \mathrm{cm}^{-3} \cdot \mathrm{c}^{-1}$ как типичных признаков начала лавинного пробоя еще не наблюдалось.

На последующей стадии $\mathbf{F}$ в период коммутации рабочего импульса параметры переходных характеристик $J_{D}(t)$ и $J_{L}(t)$, включая максимальное значение $J_{L} \approx 4.7 \kappa \mathrm{A} / \mathrm{cm}^{2}$ в момент достижения максимума $t_{2}=433$ нс, примерно совпадают для всех вариантов запуска. Это говорит о том, что в исследованных примерах при соблюдении оговоренных условий достаточности по включающему заряду РВД работали в силовой цепи как практически идеальные ключи.
Рис. 3 иллюстрирует эволюцию двумерного распределения тока в радиальных сечениях элементарных ячеек РВД на стадии $\mathbf{F}$ для всех вариантов запуска.

Интересно заметить, что для опорного режима G-0 характерна сильная локализация тока, объясняемая длительной реверсивной накачкой заряда через узкие высокопроводящие каналы катодных шунтов. Действительно, в самые ранние моменты рабочей стадии $\mathbf{F}$ амплитуда плотности тока здесь превышает 16 кА/ $\mathrm{cm}^{2}$ (рис. 3, $a$ ).

В режиме G-1 степень локализации тока резко ослабевает. Изначально локализованный в местах шунтирования заряд успевает еще за время „нереверсивной“ стадии $\mathbf{G}_{\mathbf{F}}$ перераспределиться в пространстве и включить граничащую с шунтом окрестность тиристорной части ячейки. При этом из-за противоположного действия эффектов экстракции избыточных зарядов из окрестности шунтов и преобладающего регенеративного накопления зарядов в тиристорной части ячейки максимум плотности тока перемещается в удаленные от шунта участки, достигая максимальной плотности выше $600 \mathrm{~A} / \mathrm{cm}^{2}$ при $r \approx 175$ мкм (рис. $3, b)$.

Выраженные следы первичной локализации накачки в режиме G-0 остаются даже в момент достижения максимума коммутируемого импульса тока $J(t)$ при $t=433$ нс (рис. 3,c). Наибольшая степень распределения тока по элементарной ячейке РВД для этого момента достигается в режиме запуска $\mathbf{G - 2}$, иллюстрируемого на рис. 2 кривыми $1^{\prime \prime}-3^{\prime \prime}$. Так, из рис. $3, d$ видно, что в распределении $|J(r)|$ исчезают немонотонность по $r$ и промежуточные кольцевые локализации. Перепад между значениями $|J|$ внутри основной части токового канала с площадью $0.9 S_{m}$, где $S_{m}=\pi r_{m}^{2}$, и по периметру условной границы радиусом $r \approx r_{m} / 3$ с окрестностью шунта площадью $0.1 S_{m}$ составляет всего $10 \%$ от $\left|J_{\max }\right|$.

Таким образом, полученные результаты свидетельствуют об определенных преимуществах варианта G-2 не только перед опорным G-0, но и модифицированным по идее работы [15] G-1 режимами запуска.

\section{3. Функционирование субмикросекундного РВД-модуля с управлением на фотодиодах}

Для иллюстрации важности учета инерционности конкретного управляющего ключа в РВД-коммутаторе мы выбрали пример со сценарием G-2 из разд. 2. Роль такого ключа в нем будет выполнять оптронная связка PD двух последовательных высоковольтных быстродействующих Si-фотодиодов, физические процессы в которых допускают достаточно простую одномерную интерпретацию [17,18].

Выбранная функциональная схема РВД-модуля, содержащая резонансный $L C$-накопитель энергии в контуре управляющего генератора [7], представлена на рис. 4. Она выгодно отличается от классических вариантов [2] тем, что и силовой, $C_{0}$, и управляющий, $C_{1}$, емкостные 


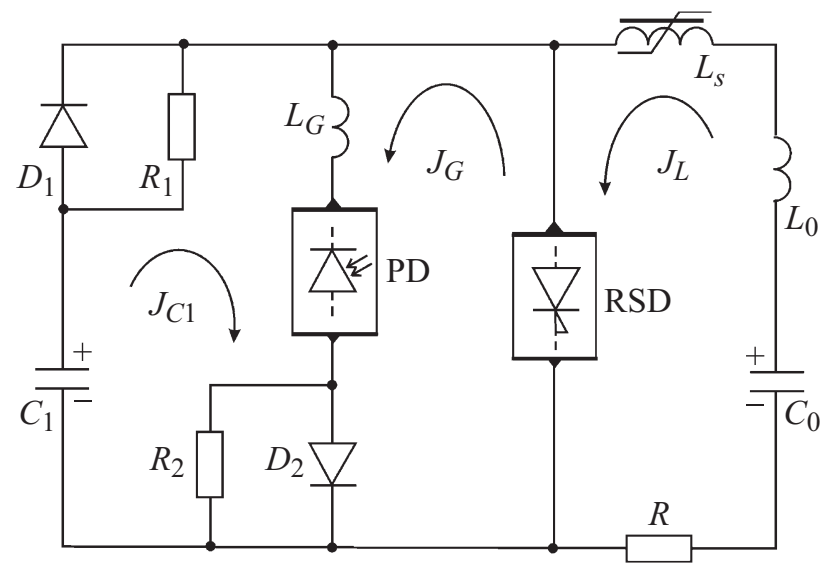

Pис. 4. Схема запуска динисторного ключа RSD в схеме РВДмодуля с оптронной управляющей связкой $\mathrm{PD}$, составленной из $\mathrm{Si}$-фотодиодов. Стрелками показаны контурные токи через цепь нагрузки $J_{L}$, оптронный ключ $J_{G}$ и накопитель управляющего контура $J_{C 1}$.

элементы накопителей заряжаются в ней до одинаковых величин и знака напряжения от единственного зарядного устройства.

$\mathrm{B}$ исходный момент времени накопители $C_{0}$ и $C_{1}$ заряжены до $U_{0}=5 \mathrm{\kappa}$, силовой дроссель размагничен, оба полупроводниковых ключа - силовой RSD и управляющий $\mathrm{PD}$ - разомкнуты и находятся под напряжением $U_{0}$, причем смещение фотодиодов отрицательно $\left(,+{ }^{*}\right.$ на катоде).

Управляемый переход ключа PD в состояние высокой проводимости осуществляется благодаря объемнораспределенной фотогенерации электронно-дырочной плазмы под действием импульсной засветки сторонним источником света.

При замыкании PD разряд накопителя $C_{1}$ на начальной стадии процесса производится током $J_{C 1}$ через резонансную часть управляющего контура ${ }_{\text {}} C_{1}-D_{1}-L_{G}-\mathrm{PD}-D_{2}$ “ с преобразованием электростатической энергии заряженного конденсатора $C_{1}$ в магнитную энергию обмотки $L_{G}$. После смены полярности смещения $C_{1}$ контурный ток $J_{C 1}$ отсекается диодами, и контур индуктивности $L_{G}$ замыкается током $J_{G}$ на контур реверсивной накачки РВД , $L_{G}-\mathrm{RSD}-D_{2}-\mathrm{PD}^{\text {“ }}$ при некотором ответвлении $J_{L s}$ в дроссель силовой цепи.

Чтобы осуществить последующее включение РВД по сценарию G-2, необходимо заблаговременно, еще до фактического завершения стадии реверсивной накачки, прервать внешнюю засветку фотодиодов в $\mathrm{PD}$ и инициировать в них весьма инерционные процессы восстановления блокирующей способности. Теперь форма импульса тока управления $J_{G}(t)$, в отличие от случаев из разд. 2, определится численно через моделируемую реакцию PD с учетом внутренних механизмов задержек нарастания и среза генерируемых импульсов.

В условиях компьютерного эксперимента желаемое согласование всех элементов конкретного РВД-модуля
Таблица 2. Параметры структуры фотодиодного элемента

\begin{tabular}{c|c|c|c|c}
\hline Тип слоя $\rightarrow$ & $n^{+}$ & $n^{\prime}$ & $p^{\prime}$ & $p^{+}$ \\
\hline$N_{n, p}^{s}, \mathrm{~cm}^{-3}$ & $10^{20}$ & $10^{17}$ & $2 \cdot 10^{15}$ & $10^{19}$ \\
$w_{n, p}$, мкм & 3 & 15 & 100 & 4
\end{tabular}

было достигнуто путем последовательной подгонки целой комбинации конструкционных и режимных параметров с учетом конкретных особенностей, вносимых оптронами.

Рабочий объем каждого фотодиода из связки PD и протекающие в нем транспортные процессы моделировались в одномерном приближении. Толщина структуры фотодиода равнялась $w=300$ мкм. Параметры диффузионных элементов ее многослойного $n^{+} n^{\prime} n_{0} p^{\prime} p^{+}$-профиля легирования заданы в табл. 2 их поверхностными концентрациями $N_{n, p}^{s}$ и толщинами $w_{n, p}$.

Для дискретных элементов силового контура схемы параметры $U_{C 0}, R, C_{0}, L_{0}$, временна́я задержка $t_{s}$ и конечная индуктивность $L_{s} 1$ дросселя выбирались такими же, как и в разд. 2. С целью усиления эффекта пополнения включающего заряда на стадии $\mathbf{G}_{\mathbf{F}}$ начальная индуктивность дросселя была снижена до $L_{s 0}=3.0 \mathrm{M \kappa} Г \mathrm{H} \cdot \mathrm{cm}^{2}$. Для управляющего контура задавались $C_{1}=1.8 \mathrm{H} \Phi / \mathrm{cm}^{2}$, $L_{G}=175 \mathrm{H} Г \mathrm{H} \cdot \mathrm{cm}^{2}, R_{1}=R_{2}=500 \mathrm{OM} \cdot \mathrm{cm}^{2}$; дискретные диоды $D_{1}$ и $D_{2}$ полагались идеальными.

Вид рассчитанных переходных зависимостей $J(t)$ и $U(t)$ представлен на рис. 5. Как и ожидалось, им присущи все качественные особенности режима G-2, установленные в предыдущем разделе и проиллюстрированные на рис. 2: разбиение интервала управления на реверсивную, $\mathbf{G}_{\mathbf{R}}$, и „нереверсивную“, $\mathbf{G}_{\mathbf{F}}$, части на кривых тока $J(t)$ и появление дополнительного пика на кривой напряжения $U(t)$, а также временны́е пропорции этого разбиения. Из-за влияния индуктивности $L_{G}$ переходный пик напряжения на связке PD

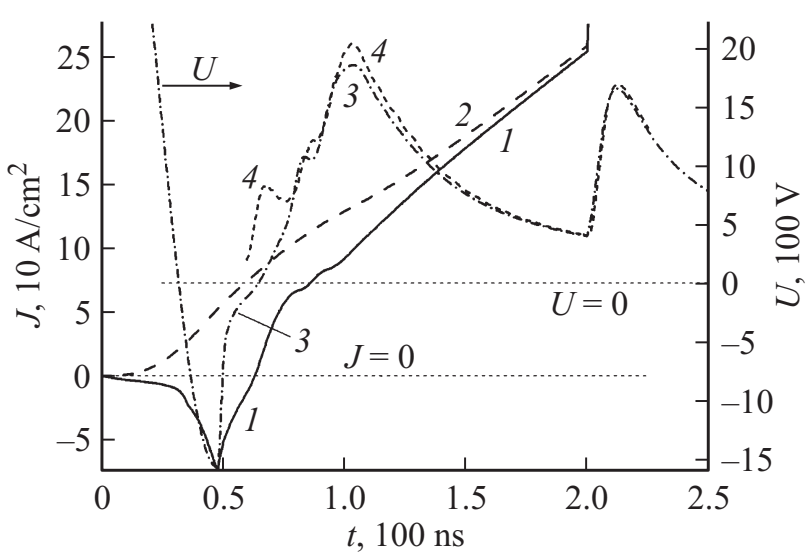

Рис. 5. Зависимости $J(t)$ и $U(t)$ при запуске РВД в схеме рис. 4.1 - суммарный ток через динистор $J_{D}, 2-$ ток через элементы цепи нагрузки $J_{L}, 3$ - напряжение $U_{A}$ на аноде RSD, 4 - напряжение $U_{\mathrm{PD}}$ на катоде оптронной связки PD. 


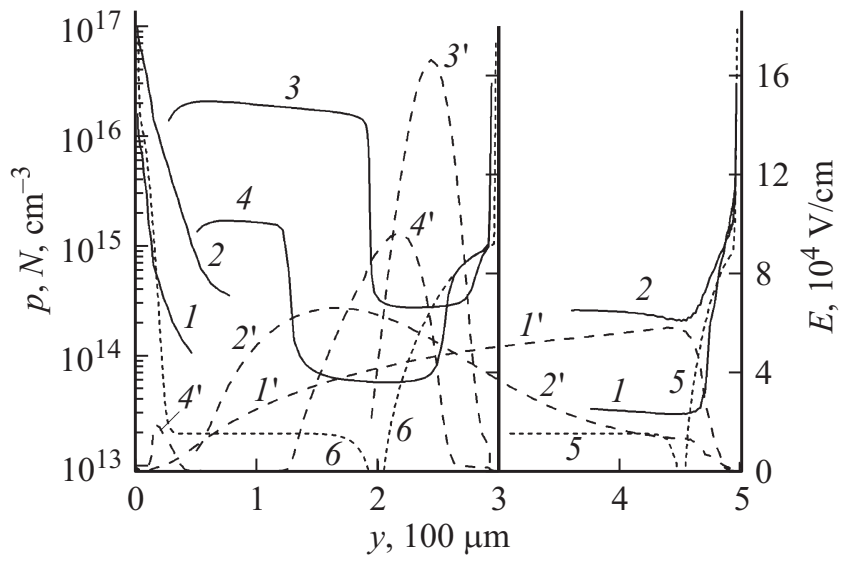

Рис. 6. Изохронные профили концентрации $p(y)(1-4)$ и поля $E(y)\left(1^{\prime}-4^{\prime}\right)$ в динисторе $\left(1,1^{\prime}, 2,2^{\prime}\right)$ и в фотодиоде $\left(3,3^{\prime}, 4,4^{\prime}\right)$ на рабочих отрезках длиной 500 и 300 мкм соответственно, в моменты времени $t_{1}\left(1,3 ; 1^{\prime}, 3^{\prime}\right)$ и $t_{2}\left(2,4 ; 2^{\prime}, 4^{\prime}\right)$ (определено в тексте). Профили примесного легирования $N(y)$ выделены пунктирными линиями 5 для динистора и 6 для фотодиода.

(кривая 4) немного превосходит напряжение на РВД (кривая 3). Небольшие осцилляции на фронте его нарастания возникают, по-видимому, по причине сложного нелинейного взаимодействия между индуктивностью $L_{G}$ контура управления и собственными индуктивностями и емкостями фотодиодов.

Особенности формы $J_{D}$ в период реверсивной накачки легко объяснить с позиции электрических процессов в управляющем контуре. Так, удлинение начальной задержки связано с инерционностью перераспределения энергии из $C_{1}$ в $L_{G}$, излом в точке максимума модуля $J_{D}$ вызывается отсечкой тока через $C_{1}$ после его разряда, быстрый рост $J_{D}$ после излома и в окрестности перехода через 0-уровень обусловлен малостью сопротивления уже частично накачанного РВД, а задержка асимптотического сближения $J_{D}$ с $J_{L}$ объясняется инерционностью восстановления блокировки оптрона.

Эволюционные перестройки концентрационно-полевых распределений в полупроводниковых элементах показаны на рис. 6 через изохронные профили распределений инжектированных дырок $p(y)$ в РВД $(1,2)$, в фотодиодах $(3,4)$ и профили поля $(1,2 ; 3,4)$ в соответствующие моменты максимумов на пиках напряжения: $t_{1}=104$ и $t_{2}=208$ нс. Видим, что для случая РВД на отрезке $0<x<500$ мкм в этом временно́м интервале имеет место качественная перестройка профиля $E(x)$ от формы с локализацией максимума в плоскости коллектора $\left(1^{\prime}\right)$ к „квазидиодной“ форме $\left(2^{\prime}\right)$ с малыми $E$ на обоих краях по вертикальной координате $y$ (по глубине структур). В эволюции профилей $p(y)$ при переходе от момента $t_{1}$ к $t_{2}$ здесь наблюдается значительное увеличение степени концентрационной модуляции инжекционного канала.

В объеме каждого из фотодиодов на отрезке $0<x<500$ мкм сначала при $t=t_{1}$ наблюдаются силь- ная локализация и рост поля в сравнительно узком слое концентрационного истощения $\left(3^{\prime}\right)$, занимающем в этот момент почти половину диффузионного слоя легирования $p^{\prime}$. Однако в точке второго максимума при $t=t_{2}$ область истощения уже охватывает половину всей толщи фотодиода, а величина $E$ при этом сильно ослабевает $\left(4^{\prime}\right)$.

Заметим, что в каждом из двух наиболее критичных (относительно возможности перенапряжения) моментов времени максимальная величина $E(x)$ остается далекой от порога начала развития лавинного пробоя как в динисторе, так и в фотодиодах.

Вернемся к обсуждению переходных характеристик РВД-коммутатора на рис. 5 и оценим достаточность величины реверсивно введенного заряда на стадии $\mathbf{G}_{\mathbf{R}}$ для успешного осуществления первичного, хотя и незавершенного, запуска РВД на „нереверсивной“ стадии $\mathbf{G}_{\mathbf{F}}$ с положительным током $J_{D}$ еще до момента насыщения дросселя. Определив приближенно по виду кривой 1 характерную скорость нарастания $J_{D} / d t$ в интервале от 50 до $200 \mathrm{нс}$ как величину $1.7 \cdot 10^{9} \mathrm{~A} \cdot \mathrm{cm}^{-2} \cdot \mathrm{c}^{-1}$ и воспользовавшись условием (1) при конкретном для данного расчета значении $w_{p}^{\mathrm{eff}}=7.5$ мкм, получим $Q_{R 0}=0.3-0.6$ мкКл/см ${ }^{2}$, что представляется в разы заниженным относительно вычисляемого по выражению (2).

Конечно, на стадии $\mathbf{G}_{\mathbf{R}}$ заряд накачки прикатодного управляющего плазменного слоя $Q_{G}^{-}$вводился в динистор через катодный шунт и был столь же сильно локализован (в качественном отношении) в окрестности шунта, как и в конце стадии накачки в опорном режиме G-0 (см. рис. 3,a). Поскольку в процессе регенеративного запуска динистора только некоторая часть величины $Q_{G}^{-}$, вычисляемой по выражениям (2), используется по назначению, то при определении „действующего“ значения заряда накачки $Q^{*}$ для использования в условии (1) вместо $Q_{R 0}$ параметр $Q_{G}^{-}$должен быть умножен на коэффициент эффективности $k_{\text {eff }}$, заведомо меньший единицы.

Ранее подобный вопрос уже возникал в задаче об эффективности неоднородно-распределенного начального избыточного заряда при включении шунтированных ячеек pnpn-структур тиристоров в критические неустойчивые возбужденные по току состояния с ничтожно малой скоростью развития неустойчивости $J_{D} / d t$ и был теоретически исследован в работе [19]. Согласно этой работе, эффективность начального заряда, сосредоточенного в окрестности шунта, является в несколько раз более низкой, чем в случае с однородным возбуждением.

Аналитической теории, описывающей неоднородный запуск тиристоров в режимы сверхбыстрого нарастания коммутируемого тока, до настоящего времени не существует. Тем не менее механизмы распространения локализованной области регенерации тока на всю ячейку в обеих ситуациях физически эквивалентны. Это дает основание при интерпретации наших расчетов использовать аргументы работы [19]. Для определен- 
ности полагая $k_{\mathrm{eff}}=0.3$, получаем, что достаточными величинами заряда первичной накачки являются скорректированные значения $Q^{*}=1-2$ мкКл/см². Заметим, что эта оценка весьма хорошо согласуется со значением $Q_{G}^{-}=1.2$ мкКл/см ${ }^{2}$, получаемым в имитационном расчете.

\section{4. Заключение}

На основе имитационного компьютерного моделирования детально изучен новый принцип включения реверсивно-включаемых динисторов в режимы со сниженным порогом первичного запуска и выполнено его сравнение по эффективности с опорным способом запуска, которым в настоящее время руководствуются разработчики РВД и высоковольтных коммутаторов на их основе. Анализ результатов численного моделирования подтверждает высокую эффективность нового способа запуска и предлагаемых нами его улучшенных вариантов, а также указывает приемлемые пути согласования конструкторских параметров субмикросекундных модификаций РВД и характеристик дискретных элементов электрических цепей, в частности параметров обостряющего дросселя силовой цепи.

При реализации нового способа в коммутационном РВД-модуле на модифицированной динисторной структуре становится реально достижимой коммутация рабочих импульсов тока с амплитудами $\sim(2-5)$ кА $/ \mathrm{cm}^{2}$ и фронтами включения 100-200нс с крутизной нарастания $d J_{A} / d t \approx(2-3) \cdot 10^{10} \mathrm{~A} \cdot \mathrm{cm}^{-2} \cdot \mathrm{c}^{-1}$ при общей длительности $\sim 500$ нс. Указанные показатели ранее рассматривались как идеализированный теоретический предел, который с большим недобором не достигается существующими коммутаторами на переключателях тиристорного типа.

Типичные величины первичного включающего заряда, вводимого импульсом реверсивной накачки из управляющего контура коммутатора и достаточного для достижения этих показателей, составляет $Q_{R 0}=1-2$ мкКл/см². В отдельных случаях, как показало моделирование, путем снижения начальной индуктивности дросселя и сокращения длительности управляющих импульсов до 10-20нс параметр $Q_{R 0}$ может быть уменьшен еще в 2-3 раза. Конечно, каждый из этих весьма привлекательных для практики случаев требует дополнительного изучения для определения допусков и гарантий по эксплуатационной области безопасной работы.

Для генерации управляющих импульсов с интенсивностью, рассчитанной на новые режимы со сниженным порогом первичного запуска, становится возможным использовать высоковольтные быстродействующие полупроводниковые генераторы пониженной мощности. Потенциальными кандидатами на роль ключей в таких генераторах могут стать подходящие модификации высоковольтных транзисторов с изолированными затворами, интегральных транзисторов [20] и тиристоров [21] с внешним полевым управлением, высоковольтные сборки $\mathrm{SiC}$-транзисторов с Шоттки-затворами и др.

Техническая возможность создания подобных субмикросекундных РВД-коммутаторов подтверждена нами в рамках полномасштабного вычислительного эксперимента для частного случая с управлением на высоковольтных фотодиодных Si-оптронах.

В заключение авторы благодарят И.В. Грехова и А.С. Кюрегяна за интерес к данному исследованию и полезные обсуждения результатов.

Работа выполнена при поддержке РНФ, номер проекта 14-29-00094.

\section{Список литературы}

[1] Г.А. Месяц. Импульсная энергетика и электроника (М., Наука, 2004).

[2] А.В. Горбатюк, И.В. Грехов, С.В. Коротков и др. А.с. СССР № 1003699 от 09.11.1982. Бюл. изобр., 1983, № 39, c. 259; ЖТФ, 52 (7), 1369 (1982); Письма ЖТФ, 8 (11), 685 (1982).

[3] В.М. Тучкевич, И.В. Грехов. Новые принципы коммутации больших мощностей полупроводниковыми приборами (Л., Наука, 1988).

[4] A.V. Gorbatyuk, I.V. Grekhov, A.V. Nalivkin. Solid-State Electron., 31 (10), 1483 (1988).

[5] M.E. Savage. IEEE Trans. Plasma Sci., 28 (5), 1451 (2000); S. Schneider, T.F. Podlesak. IEEE Trans. Plasma Sci., 28 (5), $1520(2000)$.

[6] А.В. Горбатюк. Автореф. дис. доктора физ.мат. наук (ФТИ им. А.Ф. Иоффе РАН, СПб., 2002).

[7] С.В. Коротков. ПТЭ, № 4, 5 (2002).

[8] S.V. Korotkov, A.G. Lyublinsky, Y.V. Aristov et. al. IEEE Trans. Plasma Sci., 41 (10), Pt 1, 2879 (2013). DOI: $10.1109 /$ TPS.2013.2267555.

[9] H. Wang, X. He, W. Chen, L. Xie et al. IEEE Trans. Power Electron., 29 (4), 1553 (2014).

[10] А.В. Горбатюк, И.В. Грехов, Л.С. Костина, А.В. Наливкин. Письма ЖТФ, 9(20), 1217 (1983); А.В. Наливкин. Автореф. канд. дис. (ФТИ им. А.Ф. Иоффе АН СССР, Л., 1990).

[11] С.В. Коротков, А.Л. Жмодиков. ПТЭ., № 1, 68 (2011).

[12] А.В. Горбатюк, Б.В. Иванов, И.Е. Панайотти, Ф.Б. Серков. Письма ЖТФ, 38 (8), 81 (2012).

[13] Synopsys Dev. Simulation, TCAD Sentaurus, manual. http:www.synopsys.com

[14] А.В. Горбатюк, Б.В. Иванов. ЖТФ, 85 (8), 94 (2015).

[15] А.В. Горбатюк, Б.В. Иванов. Письма ЖТФ, 41 (7), 28 (2015).

[16] А.В. Горбатюк, Д.В. Гусин, Б.В. Иванов. ФТП, 47 (3), 373 (2013).

[17] А.С. Кюрегян. ФТП, 48 (12), 1686 (2014).

[18] А.С. Кюрегян. ФТП, 49 (7), 989 (2015).

[19] А.В. Горбатюк. ФТП, 14 (7), 1364 (1980).

[20] А.С. Кюрегян, А.В. Горбатюк, Б.В. Иванов. ФТП, 50 (7), 973 (2016).

[21] И.В. Грехов, А.Л. Жмодиков, С.В. Коротков. ПТЭ, № 1, 67 (2015).

Редактор Л.В. Шаронова 


\title{
Simulation of reversely-switched dynistors in regimes with lower threshold of primary ignition
}

\author{
A.V. Gorbatyuk ${ }^{1}$, B.V. Ivanov ${ }^{2}$ \\ ${ }^{1}$ loffe Institute, \\ 194021 St. Petersburg, Russia \\ ${ }^{2}$ St. Petersburg State Electrotechnical University, \\ 197376 St. Petersburg, Russia
}

Abstract A new method for triggering reversely switched dinistors (RSDs) into submicrosecond regimes with high current rise rates at substantially lower primary ignition threshold is proposed and studied numerically in details by computer simulation. The numerical problem took into account all relevant physical laws for spatially distributed and discrete elements of RSD-modules, including nonlocal isochronous interaction between working areas of RSD or triggering photodiodes and external circuit components. Simulated results proved possible for RSD to achieve the values for current rise rates up to $d J / d t=3 \cdot 10^{10} \mathrm{~A} \cdot \mathrm{cm}^{-2} \cdot \mathrm{s}^{-1}$ in practice basing on commutators with low power semiconductor triggering switches at primary ignition levels in respect to the reversely injected charge densities of only $1-2 \mu \mathrm{C} / \mathrm{cm}^{2}$. Such numbers were considered earlier as theoretical limits unfeasible for submicrosecond-ranged thyristor-like switches. 